\title{
Sexual risk behaviors of HIV-positive persons receiving ART in Mombasa, Kenya: Longitudinal study findings
}

Stanley Luchters

Avina Sarna

Population Council

Scott Geibel

Population Council

Matthew F. Chersich

Paul Munyao

See next page for additional authors

Follow this and additional works at: https://knowledgecommons.popcouncil.org/departments_sbsr-hiv

Part of the Demography, Population, and Ecology Commons, Family, Life Course, and Society

Commons, International Public Health Commons, and the Medicine and Health Commons

How does access to this work benefit you? Let us know!

\section{Recommended Citation}

Luchters, Stanley, Avina Sarna, Scott Geibel, Matthew F. Chersich, Paul Munyao, Susan Kaai, Kishorchandra N. Mandaliya, Naomi Rutenberg, and Marleen Temmerman. 2007. "Sexual risk behaviors of HIV-positive persons receiving ART in Mombasa, Kenya: Longitudinal study findings," Horizons Research Summary. Nairobi: Population Council. 


\section{Authors}

Stanley Luchters, Avina Sarna, Scott Geibel, Matthew F. Chersich, Paul Munyao, Susan Kaai, Kishorchandra N. Mandaliya, Naomi Rutenberg, and Marleen Temmerman 


\section{Sexual Risk Behaviors of HIV-positive Persons Receiving ART in Mombasa, KENYA: LONGITUDINAL STUDY FINDINGS}

A cohort study of HIV-positive persons receiving antiretroviral therapy (ART) for 12 months revealed no increase in unsafe sex. However, there remained a risk of HIV transmission as a result of unprotected sex with an HIV-negative or unknown status partner. Safer sex counseling needs special emphasis in ART programs.

$A$ $s$ a result of the success of antiretroviral therapy (ART) in dramatically decreasing morbidity and mortality due to HIV disease, many HIV-infected persons are now living longer, healthier, and more sexually-active lives. However, unprotected sex by people living with HIV is an area of concern because of the risk of transmission to sero-discordant partners and re-infection with new, drug resistant viral strains.

There is evidence to suggest that some sero-positive individuals continue to engage in unprotected sex that place others at risk for infection and place themselves at risk for contracting secondary infections (e.g., syphilis, gonorrhea, herpes) (Crepaz et al. 2002). To learn more about the impact of ART on sexual risk behavior in a developing country setting, researchers from the Horizons Program and the International Center for Reproductive Health (ICRH) followed a cohort of HIV-positive persons receiving

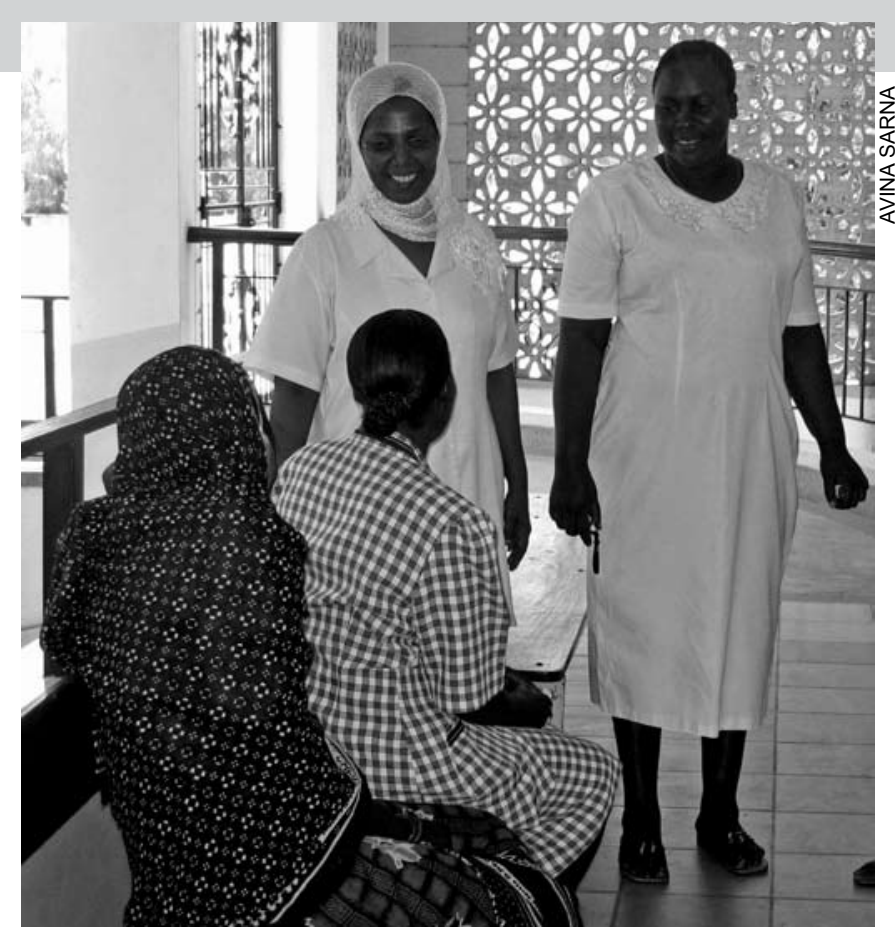

Health providers and clients at the Mkomani Bomu clinic where antiretroviral therapy is available.

ART for 12 months in Mombasa, Kenya. The study was conducted in collaboration with Coast Province General Hospital, Mkomani Bomu clinic and Port Reitz District Hospital.

\section{Methods}

A total of $234 \mathrm{HIV}$-infected, treatment naïve patients who initiated ART were enrolled in the study be-

To read more about this study, go to www.popcouncil.org/horizons/projects/Kenya_HAARTMombasa.htm
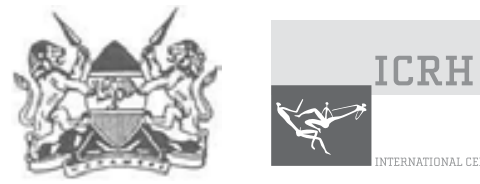
tween September 2003 and November 2004. All patients received a first line, non-nucleoside reverse transcriptase inhibitor (NNRTI) containing treatment regimen (stavudine, lamivudine and efavirenz or nevirapine) and were followed for 48 weeks. Patients were in regular contact with health workers (nurses, doctors, clinical officers, and counselors) through monthly visits at a health facility where they received routine medical care. All patients received intensive adherence counseling. Safer sex counseling was provided at the time of HIV testing and the importance of reducing sexual risk behaviours emphasized initiating ART and during follow up.

A semi-structured questionnaire was used. Interviews were conducted in English or Swahili as per patient preference prior to initiating treatment (baseline) and 12 months later (follow up). The questionnaire captured information on heterosexual and homosexual contacts with regular, non-regular and commercial sex partners; condom use; knowledge and attitudes toward condom use and ART; disclosure of HIV status to partners; HIV status of partner; and history of sexually transmitted infections (STIs). The recall reference period for most measures was 12 months at baseline and at follow-up. For analysis, unprotected sex was defined as any self-reported sex without a condom in the last 12 months with a partner known to be HIV-negative or of unknown HIV status. A regular partner was a spouse or someone with whom the respondent lived or had a stable relationship. A casual (non-regular) partner was someone the respondent had sex with only once or rarely. Partners who were paid money in exchange for sex were referred to as commercial sex partners.

Ethical approval for the study was obtained from the Kenyatta National Hospital Ethical Research Committee and the Institutional Review Board at the Population Council.

\section{Subject Recruitment and Retention}

Of the 234 participants recruited, 187 patients completed 12 months of follow up; 23 patients died; three withdrew from the study, one was incapacitated by sickness, seven transferred to other hospitals, and 10 were lost to follow-up, whereabouts unknown.

\section{Sociodemographic Characteristics}

More than half the study sample was female (64 percent); almost half (48 percent) were currently married or cohabiting while 38 percent were divorced, separated or widowed. Eightyone percent of widowed respondents were women. The mean age was 37.2 years (SD 7.2). Half of the respondents had primary education, 45 percent had secondary or higher education, and 6 percent had no education.

\section{Key Findings}

\section{Sexual activity increased marginally after 12 months of ART.}

In the year preceding ART, nearly half the respondents (48 percent) reported having had sexual intercourse. There was no difference with respect to age, sex, education and baseline CD4 counts among those who were sexually active at baseline and those who were not. Not surprisingly, sexually active respondents were more likely to be married or cohabiting than those who were not sexually active $(\mathrm{p}=0.01)$

The proportion of participants who reported sexual intercourse after 12 months on ART increased to 58 percent (Figure 1). Seventy-four respondents (40 percent) reported sex at both baseline and 12 months, 19 respondents reported sex at baseline but not at followup while 33 respondents reported sex at follow-up but not baseline. On multivariate analysis, after controlling for intra-client clustering, sex, and marital status there was no significant difference in levels of sexual activity (AOR: 1.30, 95\%CI: 0.86-1.96; $\mathrm{p}=0.21$ ).

There was no difference in sexual activity between men and women at baseline and follow up.

\section{Male respondents reporting multiple partners declined over 12 months of follow up.}

The vast majority of sexually active respondents (90 percent; 96/107) reported having a single sexual partner 
Figure 1 Self-reported sexual activity at baseline and follow up

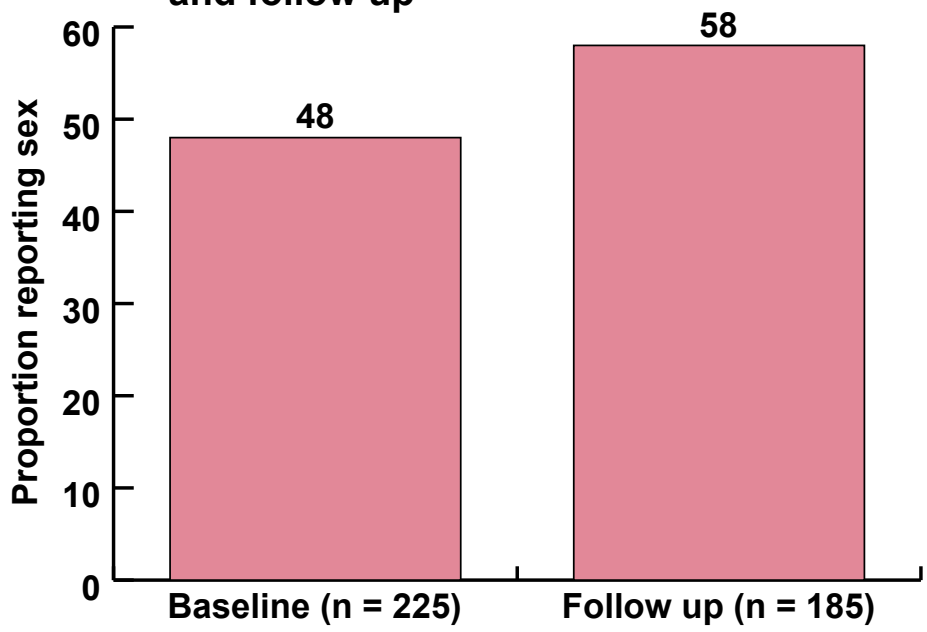

in the 12 months preceding ART. The proportion of respondents reporting a single sexual partner was higher after 12 months on ART (94 percent; 101/107) but the difference was not statistically significant $(\mathrm{p}=0.21)$. Similarly, among those who reported sex at both baseline and 12 months $(\mathrm{n}=74)$, there was a marginal, but insignificant, increase in the proportion of respondents reporting sex with a single sexual partner (89 percent vs. 93 percent; $\mathrm{p}=0.50$ ).

Prior to ART male respondents reported sex with multiple partners ( $>2$ sex partners) more frequently then female respondents ( 17 percent; $7 / 41$ vs. 6 percent, 4/66; $\mathrm{p}=0.068)$. However, at follow up a similar proportion of male (4 percent; $2 / 46$ ) and female (6 percent; 4/61) respondents reported multiple partners.

\section{Most respondents reported sex with regular partners.}

As shown in Figure 2, the vast majority of the sexually active respondents reported sex with a regular partner at baseline (98/107) and follow up (97/107). Self reported sex with casual and commercial partners decreased significantly after 12 months on ART (baseline: 15/107 vs. follow up: 10/107; p < 0.01) (see Figure 2). Among those who reported sex at both baseline and at follow up $(n=74)$, there was no change in those who reported sex with a casual or commercial partners ( $\mathrm{n}=4$ at both baseline and follow up).
Disclosure to regular partners and knowledge of regular partners'status increased over 12 months of treatment.

At baseline, just a little over half (51 percent) of the sexually active respondents reported knowing their regular partners' HIV status (HIV-positive: 40/98; HIVnegative: 10/98; unknown HIV status: 48/98). At follow up 67 percent of respondents reported knowing their regular partner's HIV status (HIV-positive: 49/97; HIVnegative: 16/97; unknown HIV status: 32/97; $\mathrm{p}<0.01$ ). Among those who reported sex at both baseline and follow up, knowledge of partner status increased significantly from 56 percent to 76 percent $(\mathrm{p}=0.003)$.

Disclosure by sexually active respondents of their HIVstatus to regular partners also increased significantly after 12 months on ART (70 percent (69/98) vs. 84 percent (82/97); $\mathrm{p}<0.05)$. Among those who reported sex at both baseline and follow up, disclosure to regular partner increased, but the difference was not statistically significant (76 percent vs. 84 percent; $\mathrm{p}=0.180$ )

\section{Unprotected sex with HIV negative or unknown status partners decreased over 12 months among sexually active respondents.}

Over three quarters (77 percent; 82/107) of the sexually active respondents reported having had unprotected sex with any partner in the 12 months preceding ART. The majority (54 patients) reported unprotected sex with HIV-negative or unknown HIV-status partners.
Figure 2 Type of partners at baseline and follow up reported by sexually active participants

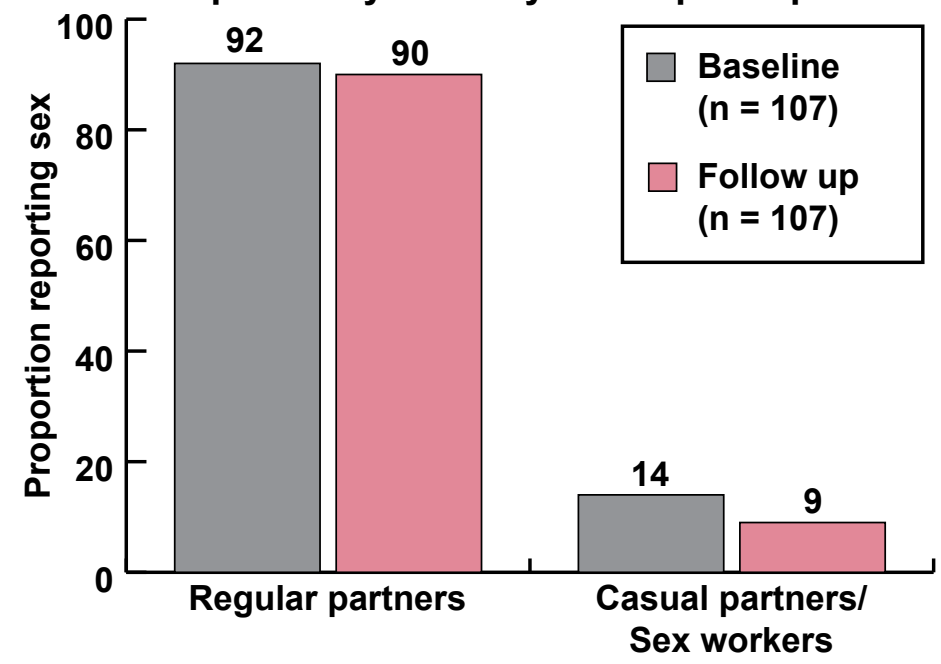


At follow up the proportion reporting unprotected sex with any partner declined to 63 percent (67/107); of whom 30 patients reported unprotected sex with HIV-negative or unknown HIV-status partners. Unprotected sex with HIV-negative or unknown status partners decreased from 50 percent $(54 / 107)$ at baseline to 28 percent (30/107) after 12 months on ART. After controlling for intra-client clustering, gender, disclosure to regular partners, and stigma, respondents were nearly half as likely at follow up to have unprotected sex with these types of partners compared to baseline (OR: 0.52; 95\% CI: 0.32-0.87; $\mathrm{p}=0.012$ ) (see Figure 3).

Risk factors associated with unprotected sex with a HIVnegative or unknown HIV-status partner were explored after 12 months on ART. After adjusting for intra client clustering, unprotected sex was associated with moderate or severe, HIV diagnosis within the past one year, not being married or cohabiting, self perceived moderate to high internalized stigma, and a body mass index of less than $18.5 \mathrm{~kg} / \mathrm{m}^{2}$. Non-disclosure of HIV-status to a regular partner was a particularly strong predictor of unsafe $\operatorname{sex}(A O R=8.3 ; 95 \%$ CI: 3.58-19.24; $\mathrm{p}<0.001)$. There was no association between respondent's gender and unprotected sex.

\section{Figure 3 Self-reported unprotected sex among sexually active participants}

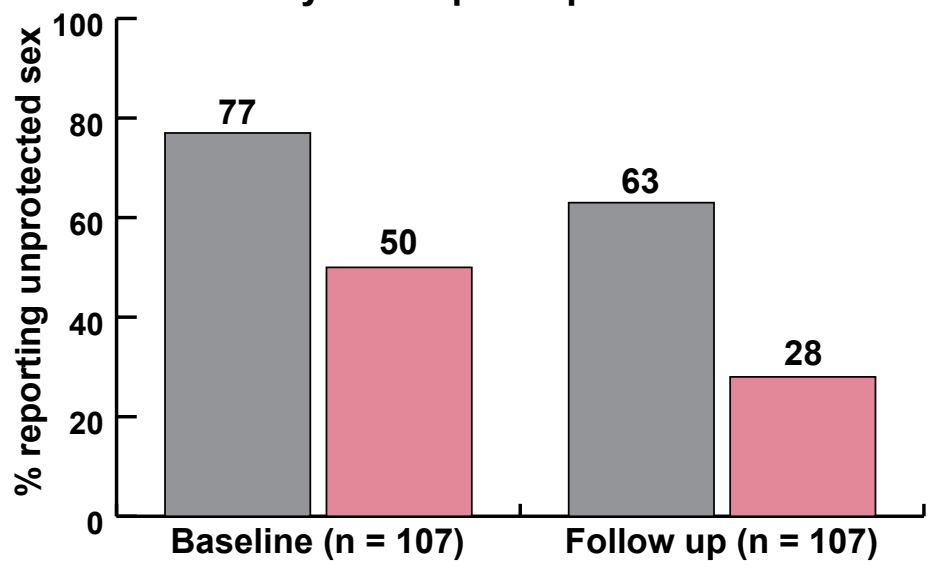
Unprotected sex
Unprotected sex with HIV- or unknown status partners

\section{Conclusions}

There have been widespread concerns of an increase in risky sexual behaviors among HIV-infected persons with improvement in health status after treatment with antiretroviral medications. The results of this study do not support such concerns. No increase in unsafe sex was noted and knowledge of partner's HIV status increased significantly. Disclosure of HIV status appears to facilitate people having safer sex.

Although the study shows no increase in risky sexual behaviors, risk of HIV transmission still remains. At follow-up, a third of respondents reported partners of unknown HIV-status and more than a fourth of sexually active participants reported unprotected sex with an HIV-negative or unknown status partner. Safer sex counseling needs special emphasis in ART programs. $\mathbb{R}$

December 2007

\section{References}

Crepaz, N. and G. Marks. 2002. "Towards an understanding of sexual risk behavior in people living with HIV: a review of social, psychological and medical findings [editorial review]," AIDS 16: 135-149.

Investigators for this study were Stanley Luchters of the International Centre for Reproductive Health, Avina Sarna and Susan Kaai of Horizons/Population Council. Intervention and study partners include Horizons, ICRH, Coast Province General Hospital, Mkomani Bomu Clinic, Port Reitz District Hospital, FHI/IMPACT, MSH/RPM Plus and Kenya Ministry of Health.

For more information on this study please contact Avina Sarna (asarna@popcouncil.org) or StanleyLuchters (Stanley.Luchters@icrh.org).

Suggested citation: Luchters, Stanley, Avina Sarna, Scott Geibel, Matthew Chersich, Paul Munyao, Susan Kaai, Kishor Mandaliya, Naomi Rutenberg, and Marleen Temmerman. 2007. "Sexual risk behaviors of HIV-positive persons receiving ART in Mombasa, Kenya: Longitudinal study findings," Horizons Research Summary. Nairobi: Population Council.

\section{Hqrizons}

Population Council/Horizons

Communications Unit

4301 Connecticut Avenue, NW

Suite 280

Washington, DC 20008

\section{Population Council}

Tel: 202-237-9400

Fax: 202-237-8410

horizons@popcouncil.org

www.popcouncil.org/horizons
This research summary is made possible by the generous support of the American people through the United States Agency for International Development (USAID) and the President's Emergency Plan for AIDS Relief under the terms of HRN-A-00-97-00012-00. The contents are the responsibility of the Horizons Program and do not necessarily reflect the views of USAID or the United States Government. 